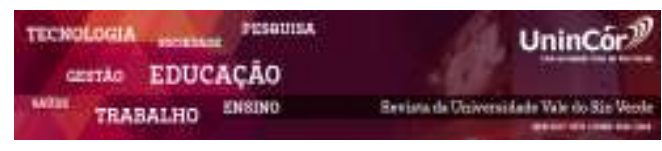

Revista da Universidade Vale do Rio Verde ISSN: 1517-0276 / EISSN: 2236-5362 Vol. 16 | n. 2 | Ano 2018

Jeferson Arantes Braz Universidade Vale do Rio Verde (UninCor) jefersonbras_1991tc@ hotmail.com

Aurivan Soares de Freitas Universidade Vale do Rio Verde (UninCor) aurivan.soares@hotmail.com

Wezer Lismar Miranda

IF Baiano - Instituto Federal Baiano wezer.miranda@ifbaiano.edu.br

Luidson Costa Silva

Universidade Vale do Rio Verde (UninCor) luidsoncs@hotmail.com

Natanael Henrique de Lacerda Universidade Vale do Rio Verde (UninCor) natanael_nhl@hotmail.com

Alexandre Tourino Mendonça Universidade Vale do Rio Verde (UninCor) alexandretourino@gmail.com

\section{DISTRIBUIÇÃO ESPACIAL DA SEVERIDADE DA BRUSONE DO TRIGO CORRELACIONADA COM A FERTILIDADE DO SOLO E PRODUÇÃO}

\section{RESUMO}

O objetivo desse trabalho foi analisar a distribuição espacial da severidade da brusone do trigo e verificar sua relação com a fertilidade do solo e a produção, utilizando a geoestatística. A área experimental compreendeu de $19.500 \mathrm{~m}^{2}$, onde foram georreferenciados 42 pontos, espaçados em malha retangular de 20 x $20 \mathrm{~m}$. Foram avaliadas em cada ponto, a fertilidade do solo, a severidade da brusone e a produção do trigo. Realizaram-se cinco avaliações da severidade da doença com auxílio de escala diagramática e os dados foram integrados em área abaixo da curva de progresso da doença (AACPD). Houve correlação negativa entre a produção e a AACPD, ou seja, o aumento da severidade da brusone influenciou a redução na produção de trigo. Os resultados desse estudo poderão ajudar a controlar a brusone de forma mais eficaz, com aplicação direcionada de fungicidas. Portanto, a geoestatística poderá contribuir na redução da contaminação humana e ambiental, e contribuir com a sustentabilidade da produção.

Palavras-chave: Pyricularia grisea. Magnaporthe grisea. Triticuma estivum. Geoestatística. Nutrição mineral.

\section{SPATIAL DISTRIBUTION OF WHEAT BLAST SEVERITY CORRELATED WITH SOIL FERTILITY AND PRODUCTION}

\begin{abstract}
This study analyzed the spatial distribution of wheat blast severity and verify its relationship with soil fertility and production using geostatistics. The experimental area comprised $19,500 \mathrm{~m}^{2}$, where 42 points were georeferenced and spaced on a regular grid $20 \mathrm{x}$ $20 \mathrm{~m}$. The wheat blast severity, soil fertility and production wheat were evaluated at each point. Five disease severity assessments were performed using a diagrammatic scale and data were integrated in the area under the disease severity progress curve (AUDSPC). There was negative correlation between production and AUDSPC, that is, the increase in the blast severity influenced the reduction in wheat production. The results of this study may help control blast more effectively, with directed application of fungicides. Therefore, geostatistics can contribute to reduce human and environmental contamination, and contribute to the sustainability of production.
\end{abstract}

Keywords: Pyricularia grisea. Magnaporthe grisea. Triticum aestivum. Geostatistics. Mineral Nutrition. 


\section{INTRODUÇÂO}

O trigo (Triticum aestivum L.) é uma planta de grande importância econômica e nutricional para a alimentação humana em todo o mundo (STEFEN et al., 2016). No Brasil, a produção de trigo não atende à demanda interna, assim, o país necessita importar essa commodity para suprir suas necessidades internas (COSTA et al., 2008). A produção de trigo está sujeita a perdas devido, principalmente, à ocorrência de várias doenças, incluindo a brusone, cujo agente etiológico é o fungo Pyricularia grisea (Cooke) Sacc. (teleomorfo: Magnaporthe grisea (Hebert) Barr (GOULART et al., 2007).

O controle da brusone tem sido realizado por meio do tratamento químico de sementes, do uso de variedades resistentes e da aplicação de fungicidas (GOULART, 1991; CRUZ et al., 2011; ROCHA et al., 2014; OLIVEIRA et al., 2015). O controle químico, além da contaminação humana e ambiental, pode ser ineficiente em função da agressividade do patógeno ou quando o trigo é cultivado em regiões com condições climáticas favoráveis a doença (CRUZ et al., 2011). Já a utilização de variedades resistentes, apesar de ser o melhor método de controle, tanto pelas vantagens do ponto de vista econômico, quanto ambiental, torna-se difícil devido à ampla variabilidade genética do patógeno e do número reduzido de fontes de resistência (ROCHA et al., 2014).

Para contribuir com o manejo de doenças, procurando reduzir o uso de fungicidas e também cultivar variedades suscetíveis, outras medidas de manejo, como o uso de plantas adequadamente nutridas, têm sido alvo de pesquisas recentes (UCHÔA et al., 2011; FREITAS et al., 2015; FREITAS et al., 2017). Em plantas com nutrição equilibrada, também se verifica rápida emissão de folhas, ocorrendo, nesta condição, lançamentos foliares em intervalos menores, além da melhoria nas barreiras de resistência física e química, pela melhor expressão da resistência, principalmente a horizontal (TAIZ; ZEIGER, 2013).

Em todo o mundo é crescente a procura por alimentos mais nutritivos e sem substâncias tóxicas, em decorrência da preocupação com a contaminação ambiental, possível de ser causada pelo uso indiscriminado de agrotóxicos. Dessa forma, há necessidade de reduzir a aplicação de produtos químicos sem comprometer a produção e a qualidade da planta.

No campo, para estudar a relação de doenças com o estado nutricional das plantas, principalmente ao longo da área de plantio ou do espaço, há a necessidade de se adotar metodologia apropriada (FREITAS et al., 2015). A estatística clássica, por não considerar a dependência espacial em muitos dos seus métodos, tem sido substituída pela geoestatística, aplicada em experimentos para gerar respostas mais condizentes com os aspectos epidemiológicos das doenças de plantas, devido ao padrão agregado do inóculo e da doença e também da variabilidade do solo ao longo da área (ALVES et al., 2006). A geoestatística faz parte do sistema de informação geográfica e representa uma das técnicas mais aplicadas no estudo de dados espacialmente relacionados (ALVES et al., 2009). A sua aplicação sofreu rápida expansão na área de epidemiologia de doenças de plantas e manejo (ALVES et al., 
2009), mais especificamente, na análise do padrão espacial para investigar fatores associados as doenças de plantas, como é caso da nutrição mineral (UCHÔA et al., 2011; FREITAS et al., 2015).

Dada a importância da brusone para a cultura do trigo e considerando a escassez de informações sobre seus aspectos epidemiológicos envolvendo a fertilidade do solo, objetivou-se com esse trabalho, estudar a distribuição espacial da severidade da brusone do trigo e verificar sua relação com a fertilidade do solo e a produção, por meio da geoestatística.

\section{MATERIAL E MÉTODOS}

O experimento foi conduzido na fazenda Roseira, em Três Corações, MG, cujas coordenadas geográficas são $21^{\circ} 37^{\prime}$ de latitude Sul e $45^{\circ} 19^{\prime}$ de longitude Oeste, com altitude aproximada de 933 metros.

O trigo da variedade BRS 208 foi cultivado em uma área de 300 hectares, com espaçamento de $17 \mathrm{~cm}$ entre linhas. A semeadura foi realizada em 06/05/2016 e a colheita em 07/09/2016. A área experimental tinha 19.500 $\mathrm{m}^{2}$, na qual foram georreferenciados com o auxílio de um receptor GPS (Global Positioning System) 42 pontos amostrais dispostos em malha regular de $20 \mathrm{~m}$ x $20 \mathrm{~m}$ (Figura 1). A cultivar BRS 208 apresenta como características resistência a oídio (Blumeria graminis tritici) e a ferrugem da folha (Puccinia recondita tritici), baixos níveis de acamamento, rendimento de grãos de $3.852 \mathrm{Kg} \mathrm{ha}^{-1} \mathrm{e}$ ciclo médio de aproximadamente 70 dias entre a emergência e o espigamento e 125 dias entre a emergência e a maturação (DOTTO et al., 2008).

Figura 1 - Malha amostral georreferenciada, utilizada para avaliar a fertilidade do solo, a severidade da brusone e a produção do trigo.

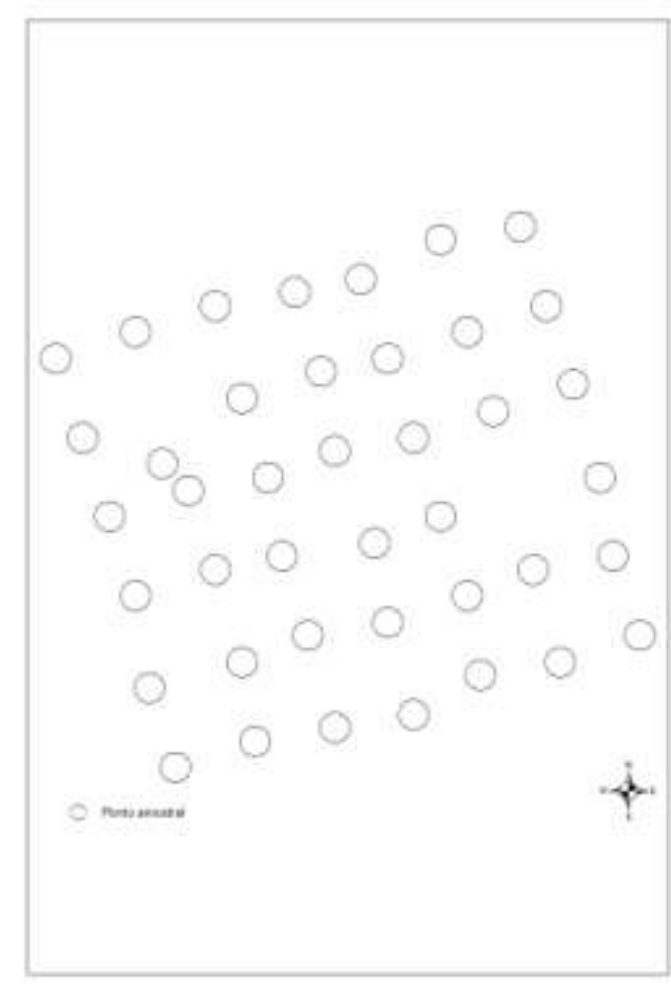

Em cada ponto amostral foi delimitada uma área de 1,0 m x 1,0 m, onde se avaliou a fertilidade do solo, a severidade da brusone e a produção do trigo.

A fertilidade do solo foi avaliada 111 dias após o plantio, por meio da coleta, com trado, de quatro amostras simples de solo, na camada de 0 a 0,20 $\mathrm{m}$ de profundidade. Após a mistura das amostras simples, foi retirada uma amostra composta com cerca de $500 \mathrm{~g}$ e enviada para o laboratório de análise de solo (Semear), da Universidade Vale do Rio Verde (UninCor), para determinar as propriedades químicas do solo.

A severidade da brusone foi avaliada em cada ponto amostral após o surgimento dos primeiros sintomas. Para isso foram selecionadas 
e marcadas 10 plantas aleatoriamente dentro da área delimitada. Foram realizadas cinco avaliações em intervalos de cinco dias com auxílio da escala diagramática proposta por Maciel et al. (2013). Os dados das avaliações foram integralizados em área abaixo da curva de progresso da doença (AACPD) de acordo com equação proposta por Shaner; Finney (1977):

$$
\mathrm{AACPD}=\sum_{i=1}^{n-1}\left(\frac{\left(y_{i}+y_{i+1}\right.}{2}\right)\left(t_{i+1}-t_{i}\right)
$$

Em que:

yi= proporção da doença na i-ésima observação;

ti= tempo, em dias, na i-ésima observação;

$\mathrm{n}=$ número total de observações.

No final do período experimental, aos 125 após o plantio, a produção do trigo foi determinada nas áreas delimitadas nos pontos amostrais. As espigas de trigo foram colhidas manualmente, descascadas e limpas. O peso foi obtido com uso de uma balança digital de precisão (Gehaka® modelo AG200).

Os dados das variáveis analisadas foram comparados por meio de correlações de Pearson, utilizando o procedimento PROC CORR no SAS.

A geoestatística foi utilizada para estudar a variabilidade espacial, bem como para escolher o modelo de semivariograma e método de ajuste que descreveu melhor a variabilidade dos dados. A dependência espacial foi avaliada com base nas pressuposições de estacionaridade da hipótese intrínseca, pela análise de semivariogramas

(BURROUGH;

MCDONNELL, 1998). A seleção dos modelos foi realizada com base nos critérios da validação cruzada, sendo escolhido, para cada variável, o modelo com erro médio mais próximo de 0 e raiz quadrada do erro médio padronizado mais próxima de 1 (WEBSTER; OLIVER, 2007a; WEBSTER; OLIVER, 2007b).

Após os ajustes dos semivariogramas, foi realizada interpolação por krigagem ordinária, a fim de estimar os valores das variáveis para locais onde as mesmas não foram medidas com base em valores adjacentes interdependentes (ALVES et al., 2006). Todas as análises geoestatísticas foram realizadas utilizando o software Arc GIS 10.1.

\section{RESULTADOS E DISCUSSÃO}

Houve correlação negativa entre a produção e a severidade da brusone do trigo em todas as avaliações e também para os dados transformadas em AACPD (Tabela 1), ou seja, o aumento da severidade da brusone influenciou a redução da produção de trigo. Segundo Goulart et al. (2007) a brusone pode proporcionar perdas de até $63,4 \%$ quando as espigas são infectadas precocemente. Isso deve-se ao fato do patógeno afetar, a formação, o tamanho e o peso específico do grão. 
Tabela 1 - Correlação entre os valores da produção de trigo, a severidade da brusone e a área abaixo da curva de progresso da doença (AACPD), no período de 5 a 25 de agosto de 2016.

\begin{tabular}{lllllll}
\hline & \multicolumn{6}{c}{ Avaliação } \\
\cline { 2 - 6 } & $05 / 08 / 2016$ & $10 / 082016$ & $15 / 08 / 2016$ & $20 / 082016$ & $25 / 08 / 2016$ & AACPD \\
\hline Produção & $-0,41^{*}$ & $-0,47^{*}$ & $-0,45^{*}$ & $-0,41^{*}$ & $-0,41^{*}$ & $-0,44^{*}$ \\
& & & & & & \\
\hline
\end{tabular}

*significativo a $5 \%$ de probabilidade

Após o teste de correlação, as variáveis significativas foram submetidas aos ajustes de modelos por meio da análise exploratória espacial. Ajustaram-se os modelos J-Bessel e exponencial para produção e AACPD, respectivamente (Figuras 2 e 3 ). De posse dos modelos ajustados, confirmou por meio dos mapas de krigagem, menor produção nas áreas com maior AACPD (Figuras 2 e 3). Dessa forma, esses resultados permitem deduzir que a brusone reduz significativamente a produção do trigo, confirmando os dados de Goulart et al. (2007). Nesse mesmo sentido, Arruda et al. (2005) evidenciaram que espigas de trigo sem sintoma visual de brusone apresentaram menores porcentagens de sementes infectadas, diferindo significativamente das sementes colhidas de espigas com sintomas da doença.

A menor produção nas áreas afetadas pela doença ocorreu em função da doença paralisar o desenvolvimento dos grãos, impedindo sua completa formação. Goulart et al. (2007) observaram que a importância econômica da brusone do trigo decorreu da redução do rendimento e da qualidade dos grãos, que, quando infectados, apresentaram-se enrugados, pequenos, deformados e com baixo peso específico.
Figura 2 - Mapa de krigagem para a produção de trigo

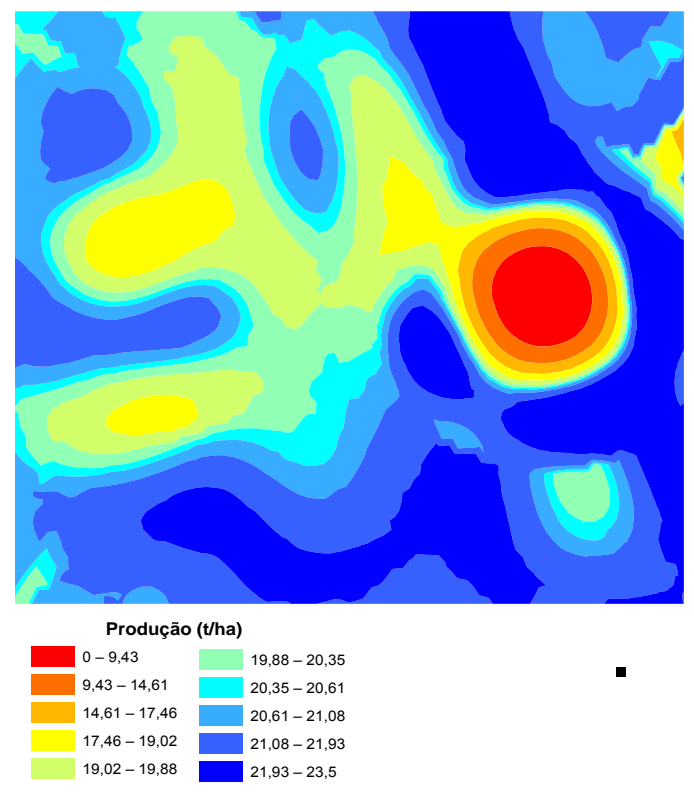

Figura 3 - Mapa de krigagem para a área abaixo da curva de progresso da doença (AACPD) da brusone do trigo.

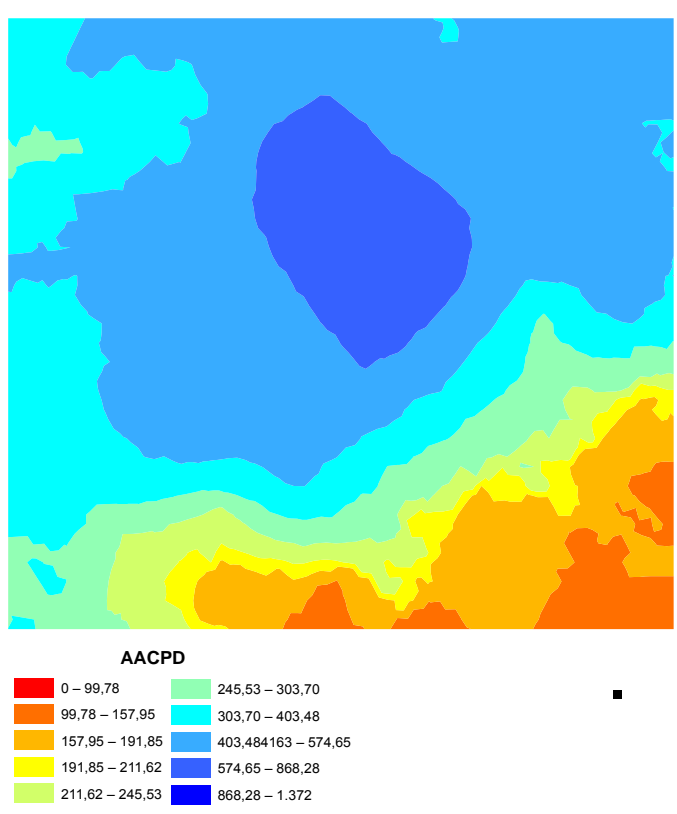


Apesar da correlação negativa entre a severidade da brusone e a produção, não houve correlação entre os dados da fertilidade do solo e essas variáveis. Porém, Freitas et al. (2015) estudando a Sigatoka-amarela (Mycosphaerella musicola) da bananeira, constataram, por meio da geoestatística, correlação positiva entre o índice de infecção da doença e os teores de areia, e negativa entre os teores de silte, matéria orgânica, soma de bases trocáveis, capacidade de troca catiônica efetiva, saturação por bases, Ca e Mg. Para esses autores, solos com maiores teores de silte e matéria orgânica apresentam maior capacidade de reter água e nutrientes, proporcionando condições favoráveis para o desenvolvimento das plantas e expressão de barreiras de resistência a doença (MARSCHNER 2012; TAIZ; ZEIGER, 2013). Em estudo realizado com a mancha marrom (Bipolaris sorokiniana) do trigo ficou comprovado que o aumento da dose de nitrogênio $(\mathrm{N})$ promoveu um acréscimo na incidência da doença nos genótipos IAC-24 e IAC-60 (TANAKA et al., 2008).

Provavelmente, não houve correlação entre os dados da fertilidade do solo e as demais variáveis, devido ao fato do solo ter sido coletado próximo ao momento da colheita. Nesse estágio, a planta pode não absorve nutrientes em razão de sua senescência ou por causa ausência

\section{REFERÊNCIAS}

ALVES, M. C.et al. Geoestatistics as methodology to study the space-time dynamics of diseases transmitted by seed-borne Colletotrichum spp. Fitopatologia Brasileira, v. 31 n. 6, p.557-563, 2006. de nutrientes no solo. O potássio $(\mathrm{K})$, por exemplo, um dos nutrientes que mais influencia a intensidade das doenças pode ser perdido por percolação ou lixiviação (DUARTE et al., 2013; FREITAS et al., 2017). Dessa forma, recomendase que nos futuros estudos, o solo seja coletado no início do aparecimento dos primeiros sintomas ou no período mais favorável ao desenvolvimento da brusone.

\section{CONCLUSÕES}

A análise espacial, utilizada nesse trabalho, permitiu realizar o diagnóstico das áreas favoráveis ao desenvolvimento da brusone e foi possível correlacionar a AACPD com a produção.

A menor produção foi encontrada nas áreas com maior AACPD.

Os resultados obtidos neste estudo poderão auxiliar agricultores, a manejar a brusone de forma mais eficaz, reduzindo a aplicação de fungicidas. Isso diminuirá o impacto ambiental, o custo de produção e contribuirá com a sustentabilidade da produção

ALVES, M. C.et al. Modeling spatial variability and pattern of rust and brown eye spot in coffee agroecosystem. Journal of Pest Science, Berlin, v. 82, n. 2, p. 137-148, 2009.

ARRUDA, M.A.et al. Reação do trigo à Magnaporthe grisea nos diferentes estádios de desenvolvimento. Fitopatologia Brasileira, v. 30, p.121-126, 2005. 
BURROUGH, P. A.; MCDONNELL, R. A. Principles of geographical information systems. New York: Oxford University Press., 1998.

COSTA, M. D. G.et al. Qualidade tecnológica de grãos e farinhas de trigo nacionais e importados. Ciência e Tecnologia de Alimentos, v. 28, n. 1, 2008.

CRUZ, M. F. A.et al. Aplicação foliar de produtos na redução da severidade da brusone do trigo. Tropical Plant Pathology, v. 36, n. 6, p.424-428, 2011.

DOTTO, S. R.et al. Cultivar de trigo BRS 208: produtividade, rusticidade e qualidade. Londrina: Embrapa Soja, 2001.

DUARTE, I. N.et al. Lixiviação de potássio proveniente do termopotássio. Pesquisa Agropecuária Tropical, v. 43, n. 2, p. 195-200, 2013.

FREITAS, A. S.et al. Spatial distribution of Yellow Sigatoka Leaf Spot correlated with soil fertility and plant nutrition. Precision Agric. v. 17, n. 1, p. 93-107, 2016.

FREITAS, A. S.et al. Interaction between potassium $(\mathrm{K})$ and calcium $(\mathrm{Ca})$ on the severity of Yellow Sigatoka in banana plants. African Journal of Agricultural Research, v. 12, n.16, p.1353-1361, 2017.

GOULART, A. C. P.et al. Danos em trigo causados pela infecção de Pyricularia grisea. Summa Phytopathologica, v. 33, n.4, p. 358-363, 2007.

GOULART, A. C. P. Avaliação de fungicidas para tratamentos de sementes de trigo (Triticum aestivum L.) no controle de Pyricularia oryzae e Helminthosporium sativum. Revista Brasileira de Sementes, Brasília, v. 13, n. 1, p. 25-29, 1991.

MACIEL, J. L. N.et al. Diagrammatic scale for the assessment of blast on wheat spikes. Summa Phytopathologica, Botucatu, v.39, n.3, p. 162-166, 2013.

MARSCHNER, H. Mineral nutrition of higher plants, 3nd ed. AcademicPres, San Diego, Calif.p.643, 2012.

OLIVEIRA, S. C.et al. Resistência cruzada aos fungicidas IQo azoxistrobina e piraclostrobina no patógeno da brusone do trigo Pyricularia oryzae no Brasil. Summa Phytopathologica, v. 41, n. 3, p. 298304, 2015.
ROCHA, J. R. A. S. C.et al. Eficiência de fungicidas no controle da brusone em trigo. Summa

Phytopathologica, v. 40, n. 4, p. 347-352, 2014.

SHANER, G.; FINNEY, R. E. The effect of nitrogen fertilization on the expression of slow-mildew inresistance in Know wheat. Phythopatology, v. 67, p. 1051-1056, 1977.

STEFEN, D. L. V.et al. A adubação nitrogenada durante o espigamento melhora a qualidade industrial do trigo (Triticum aestivum cv. Mirante) cultivado com regulador de crescimento etil-trinexapac. Revista de la Facultad de Agronomía, La Plata, v. 114, n.2, 161-169, 2016.

TAIZ, L.; ZEIGER, E. Fisiologia vegetal. 5. Ed. Porto Alegre: Artmed, 918, 2013.

TANAKA, M.A.S.et al. Incidência de doenças fúngicas e sanidade de sementes de trigo sob diferentes doses de nitrogênio e aplicação de fungicida. Summa Phytopathologica, v. 34, n. 4, p. 313-317, 2008.

UCHÔA, C. N.et al. Geostatistical modeling of blacksigatoka and relation to soil fertility. Bioscience Journal, v. 27, n. 3, p. 357-362, 2011.

WEBSTER, R.; OLIVER, M. A. Sample adequately to estimate variograms of soil properties. Journal of Soil Science, v. 43, p. 177-192, 2007a.

WEBSTER, R.; OLIVER, M. A. Geostatistics for environmental scientists. England: Wiley, $2007 \mathrm{~b}$.

Jeferson Arantes Braz

Bacharel em Agronomia pela Universidade Vale do Rio Verde UNINCOR, Três Corações Minas Gerais.

Aurivan Soares de Freitas

Mestre e doutor em Fitopatologia pela Universidade Federal de Lavras. Professor na universidade Vale do Rio Verde

\section{Wezer Lismar Miranda}

Mestre em engenharia de Água e Solo pela Universidade Federal de Lavras. Doutor em Recursos Hidricos em Sistemas Agrícolas pela Universidade Federal de Lavras. Professor no Instituto Federal Baiano

Natanael Henrique de Lacerda

Bacharel em Agronomia pela Universidade Vale do Rio Verde UNINCOR, Três Corações Minas Gerais. 
Luidson Costa Silva

Bacharel em Agronomia pela Universidade Vale do Rio Verde UNINCOR, Três Corações Minas Gerais.

\footnotetext{
Alexandre Tourino Mendonça

Mestre e doutor em Microbiologia de Alimentos pela Universidade Federal de Lavras. Graduação em Zootecnia pela Universidade Federal de Lavras. Professor na Universidade Vale do Rio Verde.
} 